Published in final edited form as:

Int Urol Nephrol. 2014 September ; 46(9): 1699-1705. doi:10.1007/s11255-014-0719-9.

\title{
Prospective evaluation of plasma kinetic bipolar resection of bladder cancer: comparison to monopolar resection and pathologic findings
}

\author{
Joseph Mashni, \\ Urology Service, Department of Surgery, Kimmel Center for Prostate and Urologic Cancers, \\ Memorial Sloan-Kettering Cancer Center, 353 East 68th Street, New York, NY 10065, USA \\ Guilherme Godoy, \\ Urology Service, Department of Surgery, Kimmel Center for Prostate and Urologic Cancers, \\ Memorial Sloan-Kettering Cancer Center, 353 East 68th Street, New York, NY 10065, USA \\ Chadwick Haarer, \\ Department of Pathology, Memorial Sloan-Kettering Cancer Center, New York, NY, USA \\ Guido Dalbagni, \\ Urology Service, Department of Surgery, Kimmel Center for Prostate and Urologic Cancers, \\ Memorial Sloan-Kettering Cancer Center, 353 East 68th Street, New York, NY 10065, USA \\ Victor E. Reuter, \\ Department of Pathology, Memorial Sloan-Kettering Cancer Center, New York, NY, USA \\ Hikmat Al Ahmadie, and \\ Department of Pathology, Memorial Sloan-Kettering Cancer Center, New York, NY, USA

\section{Bernard H. Bochner} \\ Department of Surgery, Kimmel Center for Prostate and Urologic Cancers, Memorial Sloan- \\ Kettering Cancer Center, 353 East 68th Street, New York, NY 10065, USA \\ Bernard H. Bochner: bochnerb@mskcc.org
}

\section{Abstract}

Objective-To determine whether the Gyrus ACMI plasma kinetic bipolar device (Gyrus ACMI, Southborough, MA) improves pathologic specimen preservation and clinical outcomes compared to standard monopolar electrocautery.

Patients and methods-In our prospective study, 83 patients underwent monopolar or bipolar transurethral resection of bladder tumors between April 2006 and February 2007 at Memorial Sloan-Kettering Cancer Center. Dedicated genitourinary oncology pathologists blinded to resection type and assessed pathologic features including stage and grade, presence of muscularis propria, fragment size, presence and thickness of thermal artifacts within the specimen, layer of tissue most affected, severity of tissue distortion, and diagnostic impact of thermal artifacts.

Correspondence to: Bernard H. Bochner, bochnerb@mskcc . org.

Conflict of interest The authors declare they have no conflicts of interest. 
Clinical outcomes including, perforation, obturator reflex, need for muscle paralysis, a catheter, or admission, were recorded. Clinical and pathologic outcomes between resection modality were compared.

Results-There were no significant thermal artifacts in 9/38 (23.7\%) and 11/45 (24.4\%) monopolar and bipolar specimens, respectively. The layer of bladder tissue most affected by thermal artifacts was readable in 18/38 (47.4\%) monopolar and 27/45 (60.0\%) bipolar specimens. Tissue distortion from thermal artifacts led to areas within 11/38 (28.9\%) monopolar and 7/45 $(15.6 \%)$ bipolar specimens being unreadable. Ultimately, thermal artifacts caused moderate diagnostic difficulty in 2/38 (5.3\%) specimens of the monopolar group and severe diagnostic difficulty in 1/45 (2.2\%) bipolar specimens. Clinically, there was no major difference between resection methods.

Conclusion-Plasma kinetic bipolar equipment appears to cause less tissue distortion and has the potential to facilitate staging and grading of bladder tumors. No differences in clinical outcomes were appreciated between resection methods. If these results can be repeated in larger studies, the bipolar device represents a small advancement in transurethral resection.

\section{Keywords}

Urinary bladder; Urinary bladder neoplasms; Cystoscopy; Electrosurgery; Electrocoagulation

\section{Introduction}

Since its introduction in 1910, TUR using electrocautery has been the primary technique used for the diagnosis and treatment of BTs [1]. The pathologic evaluation of TUR specimens is paramount for appropriate treatment. Correct histologic analysis of small fragments or tissues that are severely cauterized can be difficult. Additionally, evaluation is made more challenging in patients who have undergone previous resections due to scar tissue in the various layers of the bladder wall.

Traditional monopolar electrosurgical equipment requires passage of the electrical current from the loop to the grounding pad positioned on the patient's skin. Heat generated from this current facilitates the cutting of tissues but also leads to desiccation of surrounding cells. This desiccation of the tissues surrounding the resection loop often results in TAs in resected bladder tissues. TAs can obscure important characteristics of both the tumor and the normal surrounding bladder structures, limiting the pathologist's ability to provide the necessary diagnosis and proper staging for a treatment recommendation (Fig. 1).

Some centers have investigated the use of bipolar resection technologies in the management of bladder cancer [2-9]. The bipolar electrical current returns directly to the loop, rather than to the grounding pad placed on the patient's skin. These instruments have the theoretic advantage of limiting the diffusion of current and heat to the surrounding tissues. The PK technology of the Gyrus ACMI (Gyrus ACMI, Southborough, MA) device is of particular interest because of the lower voltages generated during cutting compared to standard monopolar technology, 220-320 versus 1,000-8,000 Vrms, respectively. The Gyrus ACMI PK device provides a controlled plasma pocket around the loop for cutting and sealing 
vessels. To study these potentially favorable characteristics, we performed a prospective evaluation of the Gyrus ACMI PK bipolar device, with blinded pathologic review to specifically to determine the quality specimen preservation.

\section{Patients and methods}

Following approval from our institutional review board, we evaluated pathologic and clinical features of patients who underwent TURBT with standard monopolar or Gyrus ACMI PK loop bipolar devices at Memorial Sloan-Kettering Cancer Center between April 2006 and February 2007. The data of 83 patients were evaluated for clinical outcomes, and a detailed pathologic review was completed to evaluate various features of the resected tissues.

The patients represent two consecutive series of patients, all in our contemporary experience. The bipolar specimens were all selected prospectively as our experience with the PK bipolar progressed. The monopolar specimens were selected based on the time that the specimens were obtained. We selected a series of similar size to the bipolar group, and they consisted of those monopolar cases done immediately prior to the initiation of the bipolar cohort.

The general indications for TURBT were either a previous cystoscopic examination that identified a primary or recurrent BT or a radiographic study that demonstrated a suspicious soft tissue lesion within the bladder. The procedures were performed by one of the two experienced urologists (BHB and GD) in an outpatient surgical facility at our center with either general anesthesia or monitored sedation. After an observation period in the postanesthesia care unit, patients were usually discharged from the hospital without a urinary catheter. If necessary, bladder irrigation was performed using saline irrigation fluid through a three-way catheter until it was clear of hematuria.

\section{Resection parameters}

In the monopolar group, 38 monopolar resections were performed using standard $120 \mathrm{~V}$ cutting/60 V coagulation settings and $1.5 \%$ glycine. In the PK bipolar group, 45 PK bipolar resections were performed using SP1 mode at $160 \mathrm{~V}$ cutting/80 V coagulation settings and normal saline irrigation. In both groups, a 24 French sheath with non-continuous flow was used and standard prophylactic antibiotics were administered.

\section{Pathologic review criteria}

The entire sample was evaluated histopathologically after routine processing in the same manner that a standard pathologic diagnosis is rendered. Two dedicated genitourinary pathologists ( $\mathrm{CH}$ and VER) reviewed the slides simultaneously, and a consensus opinion was rendered. One pathologist was a senior pathologist, and the other was a junior. Each specimen was assessed for various pathologic parameters including stage and grade (WHO/ ISUP_World Health Organization/International Society of Urological Pathologists 2004), presence of muscularis propria, fragment size/uniformity, presence of TA within the specimen, TA thickness, layer of tissue most affected by TA, severity of tissue distortion, and diagnostic impact of TA. TA was reported if distorted tissue was found, if it manifested 
as swelling and homogenization of connective tissue fibers, vacuolization, or alterations of the nuclei.

\section{Clinical outcomes evaluation}

The clinical parameters that were evaluated included the need for muscular relaxation to inhibit nerve reflex stimulation during the resection, perforation rate, postoperative recovery time, the need for a catheter at discharge, readmission for any reason, recatheterization either in the recovery room or subsequent to discharge, and postoperative bleeding rates. Postoperative hemoglobin and hematocrit levels, serum electrolyte, and pre- and postoperative weights were not obtained as they are not part of routine clinical care for outpatients.

\section{Results \\ Patient characteristics}

The median age for both groups was similar, 69.5 years (range 46-84 years) versus 66 years (range 27-85 years) for the monopolar and PK bipolar groups, respectively. Patients in both groups were predominantly male. Thirty of 38 patients in the monopolar group and 41 of 45 patients in the PK bipolar group had prior TURBTs. One patient in the PK bipolar group had previously undergone pelvic radiation therapy. Additionally, BCG therapy had been administered to 9 patients in the monopolar group and 11 in the PK bipolar resection group. Overall, the baseline characteristics were comparable between both groups (Table 1).

The BTs were located mostly in the posterior and lateral walls of the bladder, followed by the anterior wall, in both groups (Table 2). Distribution of the histopathologic diagnosis, stages, and grades of all specimens resected in the groups is represented in Table 3.

\section{Pathologic findings}

The monopolar and the PK bipolar groups had a similar distribution of tumor stage, specimen size, and grade. There was no sign of TA in 9/38 (23.7\%) of the specimens from the monopolar group and 11/45 (24.4\%) of the specimens in PK bipolar group. Connective tissue was the predominant segment of tissue affected by TA, this occurred in 22/38 (57.9 $\%)$ specimens of the monopolar group and in 33/44 (73.3\%) specimens of the bipolar group. The range of TA thickness was $0-1.0 \mathrm{~mm}$ in the monopolar group and $0-0.25 \mathrm{~mm}$ in the PK bipolar group. The 7 cases in which TA predominantly affected the mucosal epithelium or the tumor occurred in specimens resected by monopolar electrocautery. The muscularis propria was generally well preserved in specimens from both groups, even in areas where the connective tissue was distorted by TA (Fig. 2).

The bladder tissue layer most affected by TA was still readable in 18/38 (47.4\%) specimens from the monopolar group and in 27/45 (60.0\%) specimens from the PK bipolar group (Fig. $3)$. TA led to areas of distortion that were considered unreadable in 11/38 (28.9\%) and 6/45 (15.6\%) specimens of the monopolar and PK bipolar groups, respectively. The presence of TA in the tissues caused moderate diagnostic difficulty, which is defined as difficulty to grade and stage the lesion but still possible with immunohistochemistry, in 2/38 (5.3\%) 
specimens of the monopolar group and none in the PK bipolar group (Fig. 4). Severe diagnostic difficulty, which is defined as an inability to grade and stage a lesion, occurred in $1 / 45(2.2 \%)$ of the bipolar specimens (Table 4$)$.

\section{Clinical outcomes}

There were no significant differences in postoperative recovery time or complication rates (Table 5). While no quantitative evaluation of intraoperative bleeding was completed, qualitatively it appeared that there was less bleeding during and after the resection using the bipolar system. No difference in the need for muscle relaxation to complete the resection was identified. Obturator nerve reflexes were observed during resection of severe posteriorlateral-based tumors with both the monopolar and PK bipolar resection equipment. No bladder perforation due to nerve reflex or any other reason was observed in either of the resection groups. The proportion of patients who needed to be discharged with a Foley catheter was similar between resection groups, $7(18.4 \%)$ in the monopolar group versus 8 $(17.8 \%)$ in the PK bipolar group. One patient in the monopolar group required recatheterization due to urinary retention. All procedures were performed outpatient, and patients were discharged the same day, except for 2 patients in the PK bipolar group who required admission to the hospital to receive bladder irrigation for hematuria (Table 5).

\section{Discussion}

The objectives of performing TURBTs are threefold: (1) to obtain tissue for pathologic confirmation of histologic diagnosis, staging, and grading of BTs, (2) to achieve a complete removal of the lesion, and (3) to mitigate tumor-associated urinary symptoms such as hematuria. Since the original description of the use of monopolar cautery for fulguration of BTs by Edwin Beer, MD, in [1], improvements in cystoscope design and resection technology have been achieved. However, diagnostic difficulty and complications of resection remain clinically relevant issues. Thermal damage to tissue, whether the tissue is normal bladder wall or tumor, is a frequent finding following TURBT; this distortion of tissue architecture can limit the ability to obtain an accurate determination of stage.

There is increasingly more being published analyzing the differences between bipolar resection and monopolar resection for BTs $[5,6,9,10]$. Initial experiences were reported by Wang et al., who compared the pathologic characteristics of BT specimens resected with bipolar equipment in 11 patients with those of BT specimens resected with monopolar equipment in another 11 patients [5]. Blinded to the type of resection, the pathologist was asked to evaluate the presence of an adequate muscularis propria layer in the specimens and to score the difficulty in assigning the stage and grade of the tumors. The pathologist observed that there were no specimens with severe TAs that would make them unreadable. Furthermore, the pathologist was not able to identify the type of resection based only on the specimen characteristics. The authors concluded that bipolar resection was well suited for TURBT and that the histologic tissues sampled were of similar quality to those obtained with standard monopolar TURBT for a reliable diagnosis [5]. Lagerveld et al. [6] also reported on the results of their series of 25 tumors resected using either a standard loop resection or a bipolar angled loop wire. No differences in the depth of TA were observed 
when they compared specimens resected with either method. The authors were able to diagnose and adequately stage all specimens in their study, and they concluded that bipolar resection was a valid method for preserving BT specimens for histologic analysis. Most recently, Yang et al. [10] compared 69 bipolar to 51 monopolar TURBT; their conclusion was that there was no difference in thermal damage. It should be noted that the resection loops used were of different caliber, the patients were all left with catheters and hospitalized for approximately 4 days.

In our evaluation, specimens that were resected using PK bipolar equipment appeared similar to possibly better preserved compared to specimens resected using monopolar equipment. Though specimens were collected in a prospective and not randomized manner, pathologic evaluation was done by pathologists who were subspecialized and dedicated to only the genitourinary tract and who were blinded to resection method. When analyzing the readability of the specimens, that is the presence of no TA or TA with a degree of distortion that did not affect diagnostic reading of the tissue, $84.4 \%$ were readily interpretable within the bipolar group compared to $71.1 \%$ in the monopolar group. The only time TAs impacted diagnosis was in one specimen from the bipolar group, in which a thin swipe completely removed the lesion, but left no diagnostic tissue. This preliminary evaluation suggests that the use of the Gyrus ACMI PK bipolar device for BT resection may provide an advantage over standard monopolar electrocautery in the preservation of tissue architecture due to a decrease rate and amount of TA.

The majority of BTs can be safely resected with current monopolar technologies. However, potential complications include intra- and/or postoperative bleeding and perforation of the bladder wall. Although bleeding is commonly encountered at the time of resection, it is usually managed easily with cautery provided by monopolar generators. Postoperative bleeding leading to clot retention and requiring irrigation or fulguration is a well-recognized complication of transurethral surgery. Other potential complications include perforation of the bladder wall, which may result from aggressive, deep resection or as a result of accidental stimulation of the obturator nerve, triggering a reflex that results in violent adduction of the leg during the resection. A few centers have reported on the utilization of bipolar equipment for resection of BTs [5-8, 10, 11]. The largest series reports on $480 \mathrm{BT}$ and 376 prostate resection or incisions. With a follow-up of 12 months, the authors reported: $2 \%$ obturator nerve reflex, $0.7 \mathrm{~g} / \mathrm{dL}$ hemoglobin decline, $2.5 \%$ urethral stricture rate, and 2 $\%$ clot retention for the BT resections [8]. Recently reported, Del Rosso et al. [9] published their randomized trial comparing monopolar and bipolar resection for non-muscle invasive tumors. The main end point was to show the superiority of bipolar resection with respect to length of catheterization and hospitalization, as well as change in hemoglobin. Their results of 132 patients randomized into two groups showed no change in serum sodium or hemoglobin, but a significant decrease in catheterization and hospital stay favoring the bipolar resection: 1.3 versus 2.3 days catheterized and 2.2 versus 3.5 days hospitalized. They did briefly comment on pathological reading, mentioning that only 3 cases of histopathologic thermal artifacts were appreciated, all occurring in the monopolar group.

A major concern during bladder tumor resection is the stimulation of the obturator nerve reflex, causing perforation of the bladder. Not only does it increase the risk of bleeding and 
injury of extravesical structures, but it also carries the potential risk for tumor seeding outside the bladder into the extra- or intraperitoneal cavities. The rates of adductor contractions during TURBTs have ranged from 1.7 to $11 \%$ [12-14] but seem to be underreported in the literature if not accompanied by a serious complication. Nevertheless, because of its potential hazard in causing complications, measures should be taken to avoid the reflex to allow for complete and safe tumor resection. Initial series have shown that during PK bipolar resection, obturator nerve reflex was not observed [5, 15]; however, this has not been our experience nor that of other groups [7, 8, 11]. In a retrospective study of 121 BT specimens resected by PK bipolar equipment, Pu et al. [7] reported a $4.9 \%$ prevalence rate of adductor contraction, including one patient who had a perforation because of it. Gupta et al. [11] analyzed bipolar resection at two different energies; at the higher voltage, 3 of 10 cases resulted in obturator nerve reflex with two perforations, while at the lower voltage, no obturator nerve reflex occurred in the subsequent 98 cases. The mechanism of neurostimulation is probably dependent on multiple factors, including the location and size of the tumor, the location of the grounding pad (in monopolar instruments), the type of irrigation fluid, the degree of distention of the bladder, differences in energy levels/settings, and the experience of the surgeon. In our series, although we observed some obturator nerve reflexes during the PK bipolar resection, none resulted in a bladder perforation.

We recognize several limitations in our study. This was not a randomized study, and all specimens were obtained from one of the two experienced high-volume surgeons. Although clinical and pathologic staging characteristics were similar in each group, the nonrandomized nature of this exploratory evaluation and the relatively small number of patients in both groups limits the ability for definitive statistical analysis.

\section{Conclusions}

Histologic evaluation of bladder tumor specimens resected using standard monopolar or the PK bipolar device demonstrates that TA leading to a non-diagnostic specimen occurs in the minority of cases. This study reflects our preliminary experience with PK bipolar equipment in which $17.8 \%$ of specimens demonstrated TA that compromised readability of the specimen compared to $34.2 \%$ using monopolar resection. Any clinical benefit of bipolar resection requires confirmation from prospective studies with a larger number of patients and clinicians. If improvements in tissue preservation are confirmed, use of the PK bipolar device may represent an improvement in transurethral BT surgery. It is established that both modalities can cause an obturator reflex; however, assessment of other clinical outcomes, such as blood loss, differences in recurrence, and voiding or sexual side effects, would best be addressed in large randomized studies.

\section{Acknowledgments}

The Sidney Kimmel Center for Prostate and Urologic Cancers. This work was also supported in part by a grant from the GYRUS/ACMI Corporation. 


\section{Abbreviations}

$\begin{array}{ll}\text { BCG } & \text { Bacillus Calmette-Guérin } \\ \text { BTs } & \text { Bladder tumors } \\ \text { CBI } & \text { Continuous bladder irrigation } \\ \text { CIC } & \text { Clean intermittent catheterization } \\ \text { H\&E } & \text { Hematoxylin and eosin } \\ \text { LG } & \text { Low grade } \\ \text { HG } & \text { High grade } \\ \text { PACU } & \text { Postanesthesia care unit } \\ \text { PK } & \text { Plasma kinetic } \\ \text { PUNLMP } & \text { Papillary urothelial neoplasm of low malignant potential } \\ \text { TA } & \text { Thermal artifact } \\ \text { Tis } & \text { Carcinoma in situ } \\ \text { TUR } & \text { Transurethral resection } \\ \text { TURBT } & \text { Transurethral resection of bladder tumor } \\ \text { TURP } & \text { Transurethral resection of prostate } \\ \text { Vrms } & \text { Voltage root mean square }\end{array}$

\section{References}

1. Beer E. Removal of neoplasms of the urinary bladder. A new method, employing high-frequency (Oudin) currents through a catheterizing cystoscope. J Am Med Assoc. 1910; LIV(22):1768-1769.

2. Kriegmair M, et al. Regulated high frequency diathermy for bipolar electrocoagulation-a new method for the treatment of carcinoma of the bladder. Urol Res. 1987; 15(5):251-253. [PubMed: 3686752]

3. Lee D, Sharp VJ, Konety BR. Use of bipolar power source for transurethral resection of bladder tumor in patient with implanted pacemaker. Urology. 2005; 66(1):194. [PubMed: 15921727]

4. Rothenberger K, et al. Controlled bipolar high-frequency coagulation for transurethral application: a new method for the destruction of urinary bladder tumors. Urol Int. 1983; 38(5):257-262. [PubMed: 6636367]

5. Wang DS, et al. Use of bipolar energy for transurethral resection of bladder tumors: pathologic considerations. J Endourol. 2004; 18(6):578-582. [PubMed: 15333227]

6. Lagerveld BW, Koot RA, Smits GA. Thermal artifacts in bladder tumors following loop endoresection: electrovaporization v electrocauterization. J Endourol. 2004; 18(6):583-586. [PubMed: 15333228]

7. Pu XY, et al. Use of bipolar energy for transurethral resection of superficial bladder tumors: longterm results. J Endourol. 2008; 22(3):545-549. [PubMed: 18257673]

8. Puppo P, et al. Bipolar transurethral resection in saline (TURis): outcome and complication rates after the first 1000 cases. J Endourol. 2009; 23(7):1145-1149. [PubMed: 19530903]

9. Del Rosso A, et al. Plasmakinetic bipolar versus monopolar transurethral resection of non-muscle invasive bladder cancer: a single center randomized controlled trial. Int J Urol. 2013; 20(4):399_ 403. [PubMed: 23003110] 
10. Yang SJ, Song PH, Kim HT. Comparison of deep biopsy tissue damage from transurethral resection of bladder tumors between bipolar and monopolar devices. Korean J Urol. 2011; 52(6): 379-383. [PubMed: 21750747]

11. Gupta NP, et al. Bipolar energy for transurethral resection of bladder tumours at low-power settings: initial experience. BJU Int. 2011; 108(4):553-556. [PubMed: 21176081]

12. Collado A, et al. Early complications of endoscopic treatment for superficial bladder tumors. $\mathrm{J}$ Urol. 2000; 164(5):1529-1532. [PubMed: 11025697]

13. Kihl B, Nilson AE, Pettersson S. Thigh adductor contraction during transurethral resection of bladder tumours: evaluation of inactive electrode placement and obturator nerve topography. Scand J Urol Nephrol. 1981; 15(2):121-125. [PubMed: 7330604]

14. McKiernan JM, et al. Transurethral electrovaporization of bladder cancer. Urology. 1996; 48(2): 207-210. [PubMed: 8753730]

15. Brunken C, Qiu H, Tauber R. Transurethral resection of bladder tumours in sodium chloride solution. Urol A. 2004; 43(9):1101-1105. 


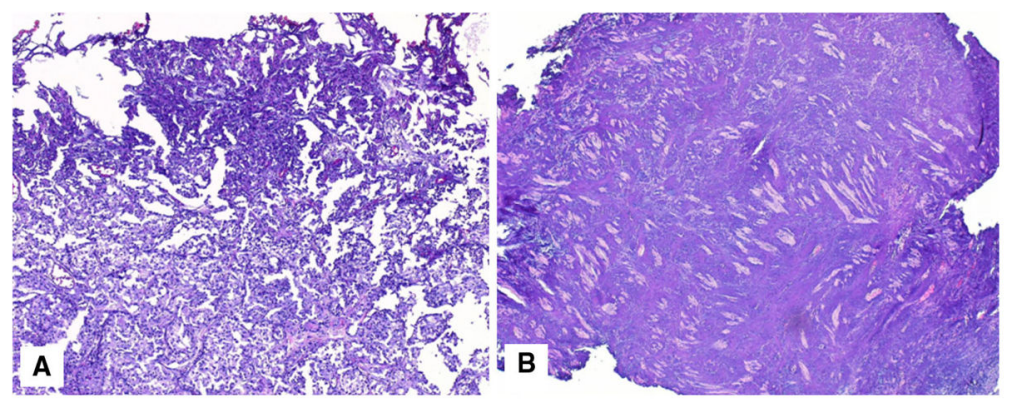

Fig. 1.

Histopathologic representation of BT specimens illustrating (a) TA-affecting tumor cells, causing moderate diagnostic difficulty, and (b) most of the sampled BT is unrecognizable due to TA (H\&E staining, original low magnification) 


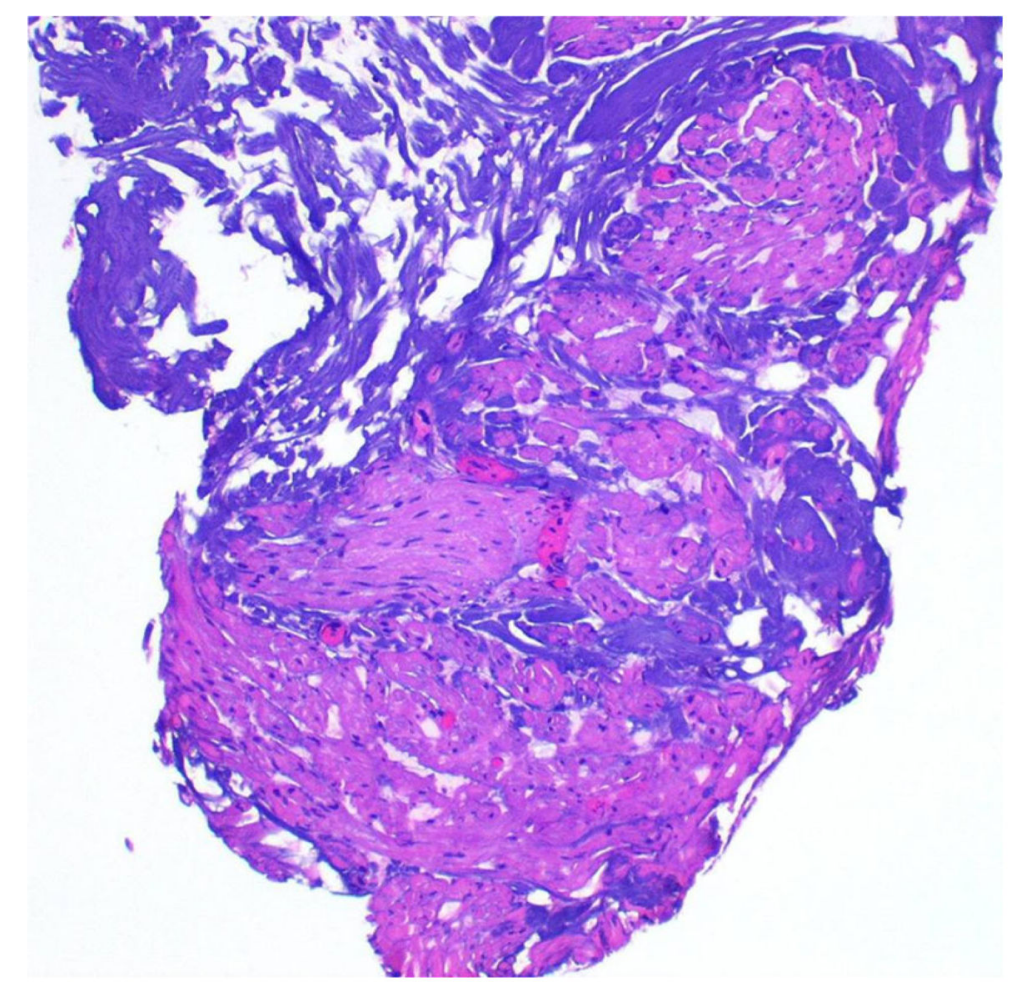

Fig. 2.

Histopathologic representation of bipolar resection BT specimen illustrating TA predominantly affecting interfascicular connective tissue with preservation of the smooth muscle (H\&E staining, original low magnification) 


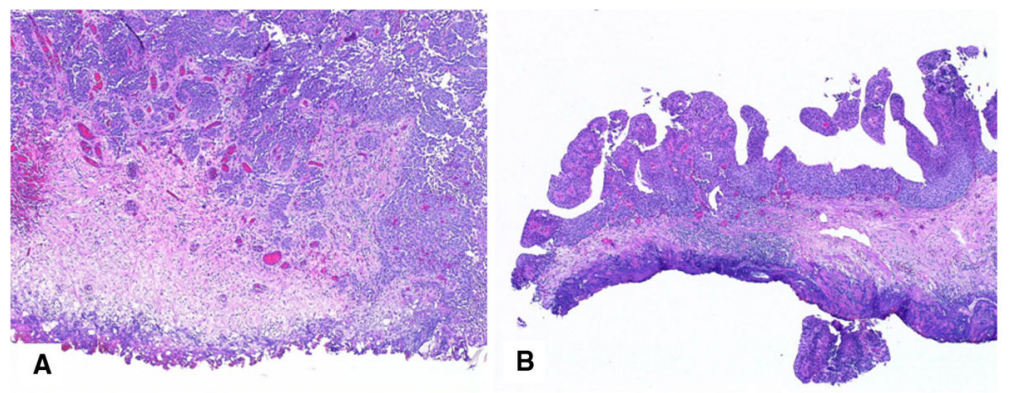

Fig. 3.

Histopathologic representation of minimal TA in BT specimens resected with either monopolar (a) or bipolar (b) instruments (H\&E staining, original low magnification) 


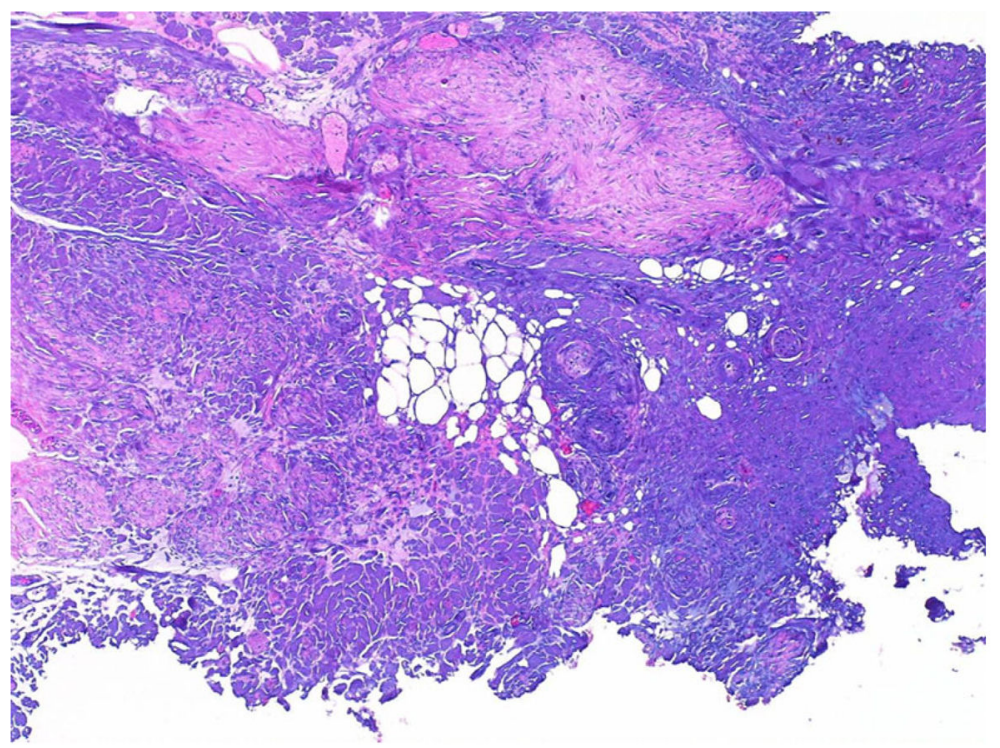

Fig. 4.

Histopathologic representation of a TA with tissue distortion caused by monopolar TURBT and resulting in moderate diagnostic difficulty. The muscularis propria is still recognizable in this specimen (H\&E staining, original low magnification) 


\section{Table 1}

Characteristics of patients undergoing monopolar or bipolar TURBT

\begin{tabular}{lll}
\hline Characteristic & Monopolar TUR & Bipolar TUR \\
\hline No. pts & 38 & 45 \\
Median age (range) & $70(46-84)$ & $66(27-85)$ \\
Gender (\%) & & \\
Males & $29(76)$ & $40(89)$ \\
Females & $9(24)$ & $5(11)$ \\
Prior TUR (\%) & $30(79)$ & $41(91)$ \\
Prior BCG (\%) & $9(24)$ & $11(24)$ \\
Dominant tumor size $(\%)$ & \\
$\quad$ 9.5 cm & $4(11)$ & $1(2)$ \\
$0.5-2$ cm & $6(16)$ & $11(24)$ \\
$2-5$ cm & $15(39)$ & $17(38)$ \\
$>5$ cm & $13(34)$ & $16(36)$ \\
Tumor no. per pt $(\%)$ & \\
Single & $21(55)$ & $25(56)$ \\
Multiple & $17(45)$ & $20(44)$ \\
2 lesions & 9 & 12 \\
3 lesions & 2 & 3 \\
4 lesions & 5 & 3 \\
5 lesions & 1 & 1 \\
6 lesions & 0 & 81 \\
Total no. of lesions & 70 & \\
\hline & &
\end{tabular}

Age in years; TUR transurethral resection, $B C G$ bacillus Calmette-Guerin 


\section{Table 2}

\section{Bladder tumor location distribution}

\begin{tabular}{lcc}
\hline Tumor location in the bladder & $\begin{array}{c}\text { Monopolar TUR } \\
\boldsymbol{n}=\mathbf{7 0}(\%)\end{array}$ & $\begin{array}{c}\text { Bipolar TUR } \\
\boldsymbol{n}=\mathbf{8 1}(\%)\end{array}$ \\
\hline Anterior wall & $14(20)$ & $12(15)$ \\
Dome & $1(1)$ & $3(4)$ \\
Posterior wall & $25(36)$ & $21(26)$ \\
Lateral walls & $19(27)$ & $26(32)$ \\
Bladder neck & $5(7)$ & $6(7)$ \\
Trigone & $6(9)$ & $9(11)$ \\
Urethra/prostate & 0 & $4(5)$ \\
\hline
\end{tabular}

TUR transurethral resection 
Table 3

Histopathologic diagnosis and staging/grading of resected bladder tumors

\begin{tabular}{lcc}
\hline Tumor location in the bladder & $\begin{array}{c}\text { Monopolar TUR } \\
\boldsymbol{n}=\mathbf{7 0}(\%)\end{array}$ & $\begin{array}{c}\text { Bipolar TUR } \\
\boldsymbol{n}=\mathbf{8 1}(\%)\end{array}$ \\
\hline Benign & $20(29)$ & $17(21)$ \\
Atypia & 0 & $1(1)$ \\
Squamous cell carcinoma & $1(1)$ & $1(1)$ \\
Adenocarcinoma & $1(1)$ & $1(1)$ \\
Urothelial carcinoma & & \\
T0 & 0 & $12(15)$ \\
PUNLMP & $4(6)$ & $1(1)$ \\
Tis & $9(13)$ & $9(11)$ \\
TaLG & $9(13)$ & $13(16)$ \\
TaHG & $17(24)$ & $9(11)$ \\
T1HG & $9(13)$ & $16(20)$ \\
T2HG & 0 & $1(1)$ \\
\hline
\end{tabular}

TUR transurethral resection, PUNLMP papillary urothelial neoplasm of low malignant potential, Tis carcinoma in situ, $L G$ low grade, $H G$ high grade 
Table 4

Effect of thermal affect on pathologic diagnosis

\begin{tabular}{lcc}
\hline & Monopolar & Bipolar \\
\hline No significant TA & $9(23.7 \%)$ & $11(24.4 \%)$ \\
Tissue with most TA; considered readable & $18(47.4 \%)$ & $27(60 \%)$ \\
Tissue with most TA; considered unreadable & $11(28.9 \%)$ & $6(15.6 \%)$ \\
TA causing moderate difficulty—readable with additional studies (IHC) & $2(5.3 \%)$ & 0 \\
Severe difficulty—inability to read & 0 & $1(2.2 \%)$ \\
\hline
\end{tabular}

$T A$ thermal affect, $I H C$ immunohistochemistry 
Table 5

Perioperative outcomes during the resection of the bladder tumors

\begin{tabular}{|c|c|c|}
\hline Outcome & $\begin{array}{l}\text { Monopolar TUR } \\
n=38\end{array}$ & $\begin{array}{l}\text { Bipolar TUR } \\
n=45\end{array}$ \\
\hline \multicolumn{3}{|l|}{ Need for muscle relaxation (\%) } \\
\hline Yes & $4(11)$ & $4(9)$ \\
\hline No & $34(89)$ & $41(91)$ \\
\hline Median time in PACU in min. (range) & $114.5(41-287)$ & $76(38-210)$ \\
\hline \multicolumn{3}{|l|}{ Need for Foley catheter (\%) } \\
\hline Yes & $7(18)$ & $8(18)$ \\
\hline No & $30(79)$ & $36(80)$ \\
\hline CIC & $1(3)$ & $1(2)$ \\
\hline \multicolumn{3}{|l|}{ Recatheterization (\%) } \\
\hline Yes $\left({ }^{*}\right.$ retention or $\S$ admitted for CBI) & $1(3)^{*}$ & $2(4)^{\S}$ \\
\hline No & $37(97)$ & $43(96)$ \\
\hline \multicolumn{3}{|l|}{ Postoperative bleeding (\%) } \\
\hline Yes (admitted for CBI) & 0 & $2(4)$ \\
\hline No & $38(100)$ & $43(96)$ \\
\hline
\end{tabular}

TUR transurethral resection, $P A C U$ postanesthesia care unit, $C I C$ clean intermittent catheterization, $C B I$ continuous bladder irrigation $*$ and $\xi_{\text {denote the type of complication }}$ 